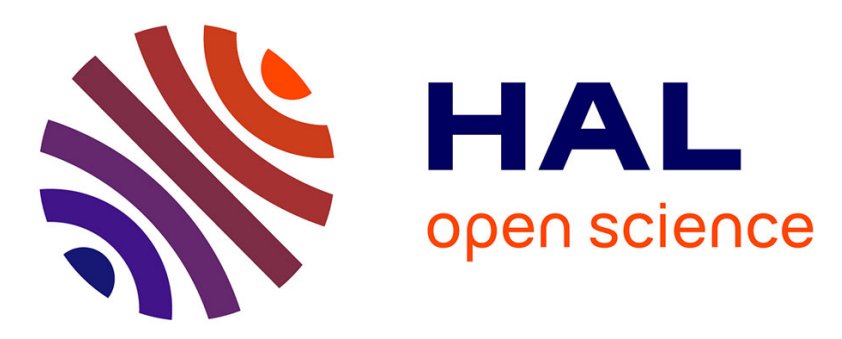

\title{
X-ray-induced radiophotodynamic therapy (RPDT) using lanthanide micelles: Beyond depth limitations
}

Slávka Kaščáková, Alexandre Giuliani, Sara Lacerda, Agnès Pallier, Pascal Mercère, Éva Tóth, Matthieu Réfrégiers

\section{- To cite this version:}

Slávka Kaščáková, Alexandre Giuliani, Sara Lacerda, Agnès Pallier, Pascal Mercère, et al.. X-rayinduced radiophotodynamic therapy (RPDT) using lanthanide micelles: Beyond depth limitations. Nano Research, 2015, 8 (7), pp.2373 - 2379. 10.1007/s12274-015-0747-5 . hal-01479428

\section{HAL Id: hal-01479428 \\ https://hal.science/hal-01479428}

Submitted on 28 Feb 2017

HAL is a multi-disciplinary open access archive for the deposit and dissemination of scientific research documents, whether they are published or not. The documents may come from teaching and research institutions in France or abroad, or from public or private research centers.
L'archive ouverte pluridisciplinaire HAL, est destinée au dépôt et à la diffusion de documents scientifiques de niveau recherche, publiés ou non, émanant des établissements d'enseignement et de recherche français ou étrangers, des laboratoires publics ou privés. 


\section{TABLE OF CONTENTS (TOC)}

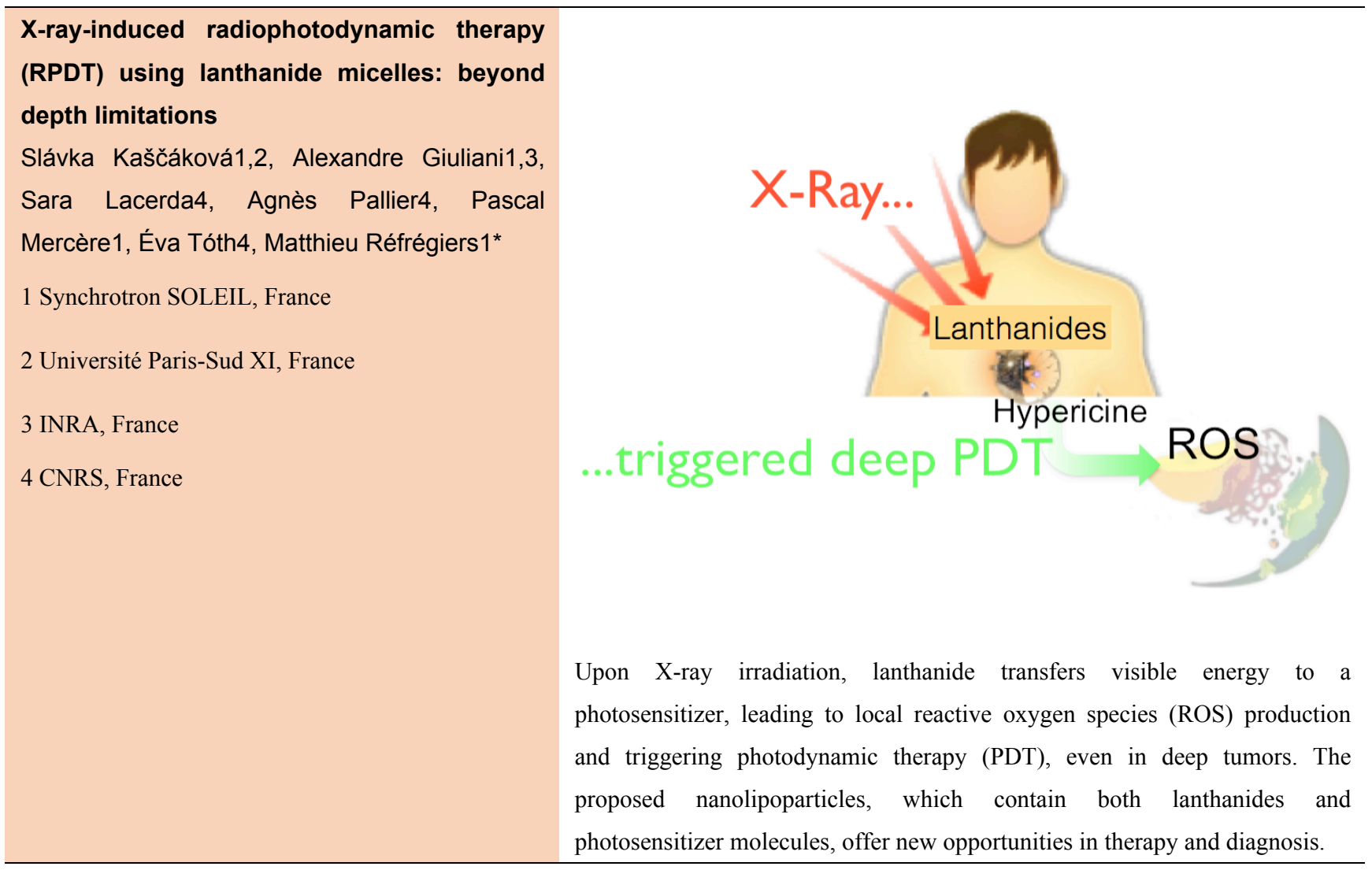

Provide the authors' website if possible.

Matthieu Réfrégiers, http://www.synchrotron-soleil.fr/portal/page/portal/Recherche/LignesLumiere/DISCO 



\title{
X-ray-induced radiophotodynamic therapy (RPDT) using lanthanide micelles: Beyond depth limitations
}

\author{
Slávka Kaščáková ${ }^{1,2}$, Alexandre Giuliani ${ }^{1,3}$, Sara Lacerda ${ }^{4}$, Agnès Pallier $^{4}$, Pascal Mercère $^{1}$, Éva Tóth $^{4}$, \\ Matthieu Réfrégiers ${ }^{1}(\varangle)$ \\ ${ }^{1}$ Synchrotron SOLEIL, L'Orme des Merisiers, 91190 Gif-sur-Yvette, France \\ ${ }^{2}$ Université Paris-Sud XI, UMR-785, 94807 Villejuif, France \\ ${ }^{3}$ INRA, UAR1008 Caractérisation et Élaboration des Produits Issus de l'Agriculture, 44316 Nantes, France \\ ${ }^{4}$ Centre de Biophysique Moléculaire, CNRS, Rue Charles Sadron, 45071 Orléans Cedex 2, France
}

\author{
Received: 24 December 2014 \\ Revised: 3 February 2015 \\ Accepted: 10 February 2015 \\ (C) Tsinghua University Press \\ and Springer-Verlag Berlin \\ Heidelberg 2015

\section{KEYWORDS} \\ photodynamic therapy, \\ liponanoparticles, X-Ray, \\ deep tumour, \\ photosensitizer
}

\begin{abstract}
We report lanthanide-based micelles integrating hypericin (Hyp) for $X$-ray-triggered photodynamic therapy (PDT). The lanthanide luminescence induced by X-ray irradiation excites the photosensitizer, which leads to the generation of singlet oxygen. This versatile approach can be extended to other photosensitizers or other types of liponanoparticles and can allow for magnetic resonance imaging (MRI) guidance.
\end{abstract}

\section{Introduction}

Over the past several decades, nanoparticles have attracted growing interest as imaging probes [1-6] and drug carriers [7-9]. More recently, advances in theranostics [10] have led to the development of nanocarriers that combine diagnosis, drug delivery, and therapy monitoring. Nanoparticles loaded with lanthanides, in particular Gd(III), have been explored as contrast agents in magnetic resonance imaging (MRI) [11-13], and they have also been used as radiosensitizers in neutron capture therapy (NCT) [14]. The unique photophysical features of luminescent lanthanides, such as large Stokes shifts (avoiding concentration-dependent self-absorption problems), long luminescence lifetimes, narrow, non-overlapping emission bands (enabling the simultaneous use of

Address correspondence to matthieu.refregiers@synchrotron-soleil.fr

(A) TSINGERSTY HARESS Springer 
multiple Ln probes) as well as characteristic emission insensitivity to environmental changes, make them specifically useful for cellular optical imaging $[2,15$, 16]. Rare earths also exhibit a unique property known as X-ray excited optical luminescence (XEOL), pioneered by Urbain in 1909 [17], which refers to the luminescence of a light-emitting material upon absorption of an energetic X-ray photon.

In this study, we propose to exploit XEOL of lanthanide-containing liponanoparticles and use the locally generated light for photodynamic therapy (PDT). To trigger the production of reactive oxygen species, PDT requires the simultaneous presence of three elements at the same location, namely the photosensitizer, oxygen, and electromagnetic radiation [18]. However, PDT is often impeded by the absorption maximum of the photosensitizer, which falls outside the tissue optical window $(700-1,100 \mathrm{~nm})$, where most tissue chromophores, including oxy- and deoxy-hemoglobin, melanin, and fat, have only weak absorption. The most straightforward method for overcoming this problem involves the creation of sensitizers with bathochromically shifted absorption maxima. Toward this objective, Roelants et al. synthesized hypericin (Hyp) derivatives [19]. Although the photoabsorption of Hyp could be tuned and shifted to longer wavelengths, its photosensitizer activity was unfavorably affected.

A fundamentally different approach is to circumvent external ultraviolet-visible (UV-VIS) excitation by using a locally generated light source in the tissue for PDT. Carpenter et al. [20] have demonstrated that chemiluminescence produced from the reaction of luciferin with luciferase and molecular oxygen could yield sufficiently intense and long-lived emission for exciting Hyp [21, 22]. Despite the promising in vitro results, in vivo applications were hampered by the delivery of the chemiluminescence components and/or the viral vectors required to transfect the target cells with the luciferase gene.

The X-ray excited optical luminescence of lanthanides appears as an alternative to the local light source for
PDT. Wang et al. considered possible applications of scintillation nanoparticles for biological purposes [23] and proposed $\mathrm{Tb}^{3+}$-doped $\mathrm{LaF}_{3}$ nanocrystals [24] conjugated with the photosensitizer meso-tetra(4-carboxyphenyl) porphine for PDT [25]. Very recently, a porphyrin-conjugated $\mathrm{Tb}_{2} \mathrm{O}_{3}$ nanoparticle has been evaluated for singlet oxygen production following X-ray irradiation [26]. In addition to lanthanide-based compounds, copper-cysteamine complex nanoparticles [27] as well as copper and cobalt co-doped $\mathrm{ZnS}$ [28] have also been studied in this context.

2 Results and discussion

In this communication, we report on a micellar system for X-ray-triggered PDT. The micelles consist of amphiphilic lanthanide chelates and incorporate Hyp as photosensitizer in their hydrophobic core (Fig. 1). Similar micellar assemblies, formed by $\mathrm{LnC} 12$ complexes, have been previously shown to encompass chromophores that can efficiently sensitize lanthanide luminescence [29]. The non-toxicity of the pyridinic $\mathrm{Ln}^{3+}$ complex has been previously demonstrated in mice [30]. The micellar structure offers an easy way to integrate highly hydrophobic molecules such as typical photosensitizers. Liponanoparticles could also be interesting carriers for delivering a high payload of the photosensitizer to sites of interest, which could not be otherwise achieved owing to their poor water solubility. We hypothesized that X-ray irradiation of these micelles can trigger the cascade of events specific to PDT resulting in reactive oxygen species (ROS) production. Moreover, local light generation via X-ray excitation of the lanthanide could allow for deep cancer treatment with PDT that could be synergistically combined with classical radiotherapy. Additionally, the presence of lanthanides in the micellar structure offers optical or magnetic resonance imaging capabilities, either at the cellular level or in vivo, respectively. 


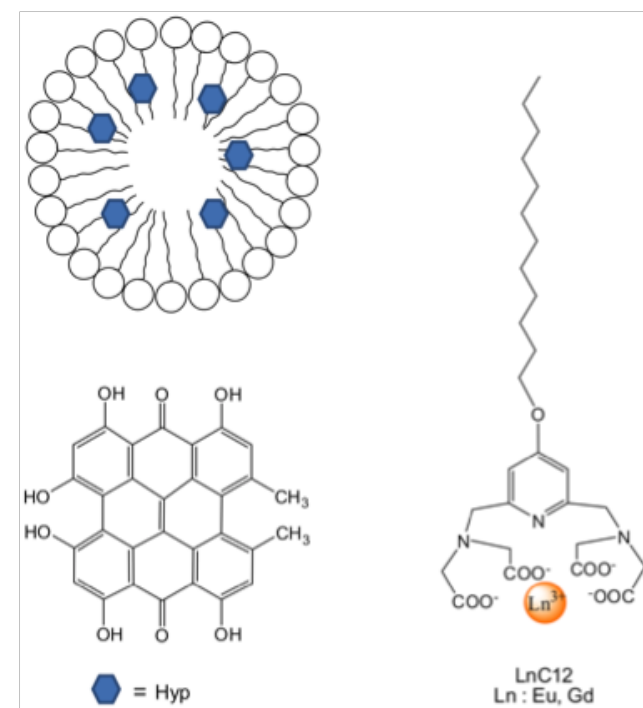

Figure 1 Schematic representation of the studied Hyp-GdEuC12 micellar particles and the respective structures of the amphiphilic LnC12 complex and the photosensitizer Hyp.

While the absorption and emission spectra for most lanthanides have been widely reported [2, 23, 31, 32], data on direct X-ray-excited luminescence of lanthanides are rather scarce [33]. Figure 2 shows the luminescence pattern of $\mathrm{Eu}^{3+}$ and $\mathrm{Gd}^{3+}$ in aqueous solution in the VIS-UV region after X-ray excitation. In Fig. 2(a), $\mathrm{Eu}^{3+}$ exhibits emission peaks at 595, 618, and $700 \mathrm{~nm}$, while $\mathrm{Gd}^{3+}$ shows an emission pattern in the UV region (Fig. 2(b)). The relative luminescence intensity is proportional to the lanthanide concentration (Figure $\mathrm{S} 1$ in the Electronic Supplementary Material (ESM)). The emission spectra following X-ray excitation (Fig. 2) are in full agreement with the photoluminescence spectra presented in Fig. S2 in the ESM.
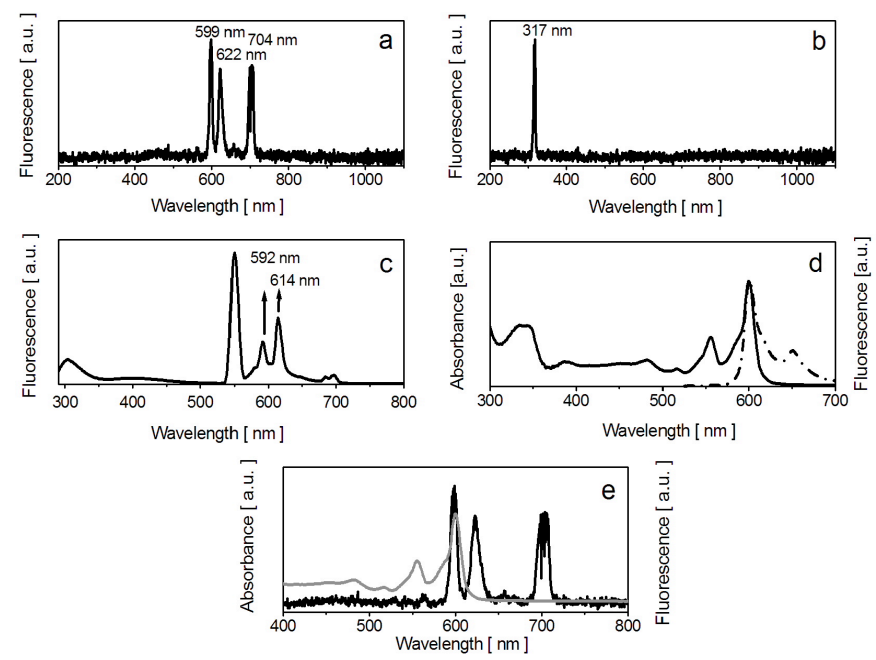

Figure 2 X-ray-induced luminescence of (a) $\mathrm{EuCl}_{3}(96 \mathrm{mM})$ and (b) $\mathrm{GdCl}_{3}(199 \mathrm{mM}$ ) in aqueous solution. (c) Luminescence of the GdEuC12 micelles in aqueous solution following 275-nm excitation. Note: The peak at $550 \mathrm{~nm}$ corresponds to second harmonic excitation. (d) Absorption spectrum (solid line) and fluorescence emission (dashed line) after 51-nm excitation of 1 $\mu \mathrm{M}$ Hyp in DMSO. (e) Overlay between the luminescence spectrum of $\mathrm{EuCl}_{3}$ (black line) and the absorption spectrum of Hyp (gray line) highlighting the level of donor-acceptor overlap.

The photophysical properties of the micellar system were first studied following UV excitation, which corresponds to the excitation of the pyridine moiety of the ligand. Pyridine has been previously shown to be a good sensitizer of europium luminescence [29]. Upon excitation at $275 \mathrm{~nm}$, the GdEuC12 micelles (without Hyp) present emission peaks at 595 and $618 \mathrm{~nm}$ (Fig. 2(c)), corresponding to characteristic Eu emissions. The characteristic fluorescence of $\mathrm{Gd}$ at $317 \mathrm{~nm}$ within the $\mathrm{GdEuC} 12$ micelles is not detectable, which could be indicative of an energy transfer from Gd to $\mathrm{Eu}$ upon excitation at the $275-\mathrm{nm}$ wavelength. To achieve an efficient energy transfer to the photosensitizer, the emission spectra of $\operatorname{Ln}$ (III) should overlap with the excitation spectra of Hyp. As Fig. 2(d) demonstrates, the absorption (and emission) spectra of Hyp nicely agree with the emission spectrum of the 
micelles (Fig. 2(c)), which can thus afford an efficient energy transfer from the lanthanide to the photosensitizer.

To test whether energy transfer indeed occurs in the micelles between $\mathrm{Eu}^{3+}$ and Hyp, micelles were prepared by incorporating different concentrations of Hyp (the photoluminescence spectra of Hyp-GdEuC12 are presented in Fig. S3 in the ESM), and the luminescence spectra in the visible region were recorded after X-ray excitation (Fig. 3(a)). As Fig. 3(a) shows, upon X-ray irradiation of the Hyp-GdEuC12 micelles, we detect characteristic $\mathrm{Eu}^{3+}$ luminescence in the visible spectral region. On the other hand, we do not observe the luminescence emission of Hyp (at 601 $\mathrm{nm}$ ) because it is below the detection threshold of our spectrometer. However, Fig. 3(a) demonstrates a clear decrease in the europium luminescence when the Hyp concentration in the micelles increases. Moreover, the observation that europium luminescence decreases with increasing Hyp concentration in the micelles gives credence to an energy transfer to Hyp from $\mathrm{Eu}(\mathrm{III})$ following $\mathrm{X}$-ray excitation of the lanthanide. Such an energy transfer is a prerequisite for ROS production from the lanthanide-photosensitizer association, such as singlet oxygen. Therefore, to obtain prove that our micellar system could be an efficient agent for PDT, we have measured the singlet oxygen generated by Hyp following X-ray absorption of the lanthanide ion. The singlet oxygen production was monitored by mass spectrometry using methoxyvinylpyrene (MVP) as a singlet oxygen probe [34]. Upon X-ray irradiation, the abundance of 1-pyrenecarboxaldehyde, which is the product of the reaction between MVP and singlet oxygen, increases with increasing Hyp concentration in the Hyp-GdEuC12 micelles (Fig. 3(b)). In contrast, only a negligible amount of 1-pyrenecarboxaldehyde is detected in the non-irradiated sample even for the highest Hyp payload. The correlation between the amount of Hyp and the production of singlet oxygen clearly shows that, upon X-ray absorption, energy transfer occurs from the lanthanide to the photosensitizer, which then triggers the PDT cascade of events.
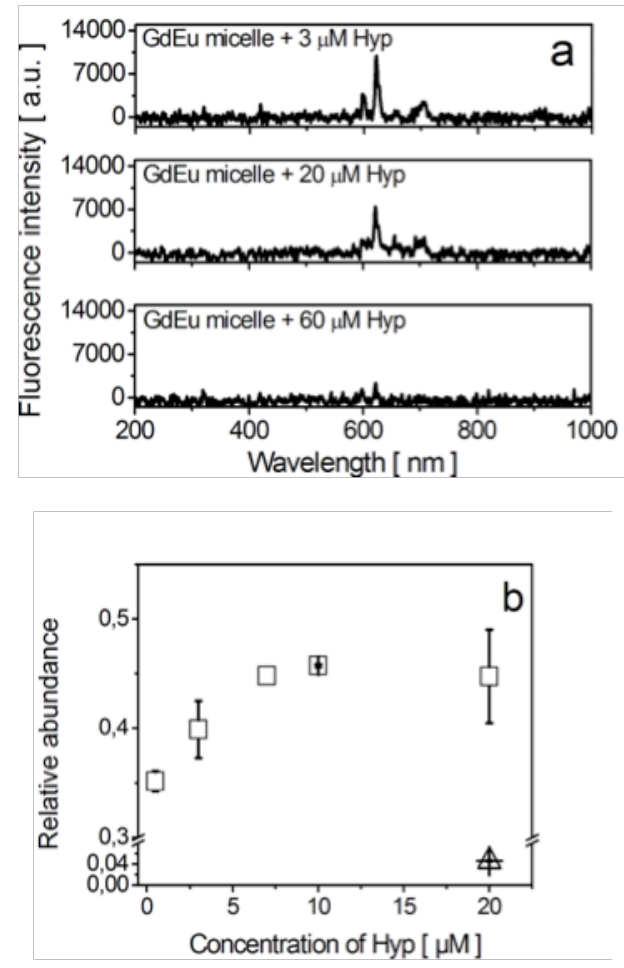

Figure 3 (a) The X-ray-induced luminescence of GdEuC12 micelles in the presence of increasing concentrations of Hyp. (b) Singlet oxygen production as indicated by the abundance of 1-pyrenecarboxaldehyde, measured by mass spectrometry, for non-irradiated (triangle) and irradiated (square) Hyp-GdEuC12 micelles.

The UV luminescence properties of the micellar assembly have been used to follow the localization of nanoparticles in cells. Figure 4 presents visible and fluorescence images of HeLa cells treated with the micelles. Fluorescence excitation under $395 \mathrm{~nm}$ provides a good contrast for lanthanides. Interestingly, cells treated with Hyp-GdEuC12 micelles exhibited a strong fluorescence located in the nucleus (Fig. 4(h)) in contrast to those treated with $\mathrm{GdEuC12}$ micelles (Fig. 4(d)) or with Hyp (Fig. 4(f)). Obviously, the system targets the nucleus of the cells, which is an important characteristic for both radiotherapy and PDT. 

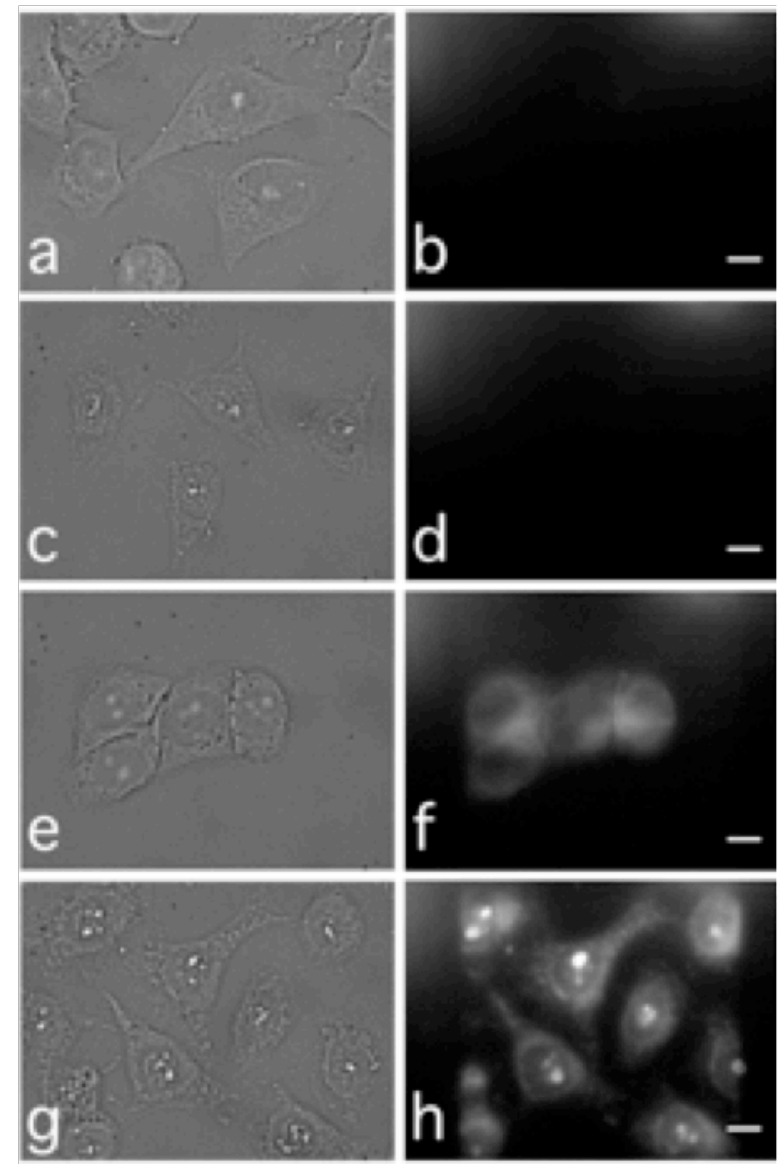

Figure 4 The HeLa cells (transmission images in left column) were incubated with various concentrations of Hyp and GdEuC12 before fluorescence imaging (right column) with 395-nm excitation (chosen for better contrast of $\mathrm{GdEu}$ fluorescence excitation compared to Hyp excitation). Control cells (a) and (b) and cells treated only with $500 \mu \mathrm{M}$ GdEuC12 (c) and (d) do not present any fluorescence. Cells treated only with $4 \mu \mathrm{M}$ Hyp (e) and (f) show the typical localization of Hyp, surrounding the nucleus. Cells treated with and Hyp-GdEuC12 (500 $\mu \mathrm{M}$ Ln, $4 \mu \mathrm{M}$ Hyp (g) and (h) present strong fluorescence associated to the nucleoli. The scale bar is $20 \mu \mathrm{m}$.

\section{Experimental}

The preparation of GdEuC12 and Hyp-GdEuC12 micelles is summarized in the ESM. The incorporation of Hyp into the hydrophobic core of the micelle was verified by absorption and fluorescence measurements of Hyp as previously described [35].

Fluorescence spectra were recorded at $20{ }^{\circ} \mathrm{C}$ using a FluoroMax-4 (HORIBA Jobin-Yvon INC, Chilly Mazarin, France) spectrofluorimeter. A quartz cuvette with a $1-\mathrm{cm}$ pathlength was used for measurement. To record the excitation and emission spectra of individual compounds, the best excitation and emission wavelengths were chosen after optimization (see Fig. 4 caption). Fluorescence spectra were treated under Microcal Origin, version 8.0 (Microcal Software, Inc., Northampton, MA).

The X-ray irradiation was performed on the METROLOGY and Tests beamline of the Synchrotron SOLEIL (see the ESM).

Singlet oxygen production after X-ray irradiation of Hyp-GdEuC12 micelle solutions was detected by mass spectrometry using methoxyvinylpyrene (MVP) as a singlet oxygen probe [34] (see ESM).

In vitro imaging was performed on the Disco beamline [36] using the microscope [37] controlled under $\mu$ Manager [38]. The excitation came from a synchrotron bending magnet and was monochromatised at $395 \mathrm{~nm}$. A 400-nm dichroic mirror reflected the light toward the cells. Emission was filtered through a long-pass 565 -nm filter.

\section{Conclusions}

In summary, we report here a liponanoparticle construct based on $\mathrm{GdEuC12}$ micelles incorporating Hyp as a photosensitizer in their hydrophobic core, which provides X-ray-induced singlet oxygen production for PDT. Our approach is highly versatile and can be translated to other photosensitizers and to other types of liponanoparticles such as liposomes that are already widely used in medical applications and can be easily adapted to various biological targets. This demonstration of X-ray-induced photodynamic therapy using lanthanide complexes represents an important breakthrough in PDT. The local excitation of the photosensitizer by X-ray-induced luminescence allows going beyond the existing boundaries of PDT, which is traditionally limited to superficial areas. This novel class of PDT sensitizers becomes suitable for 
treating deep-seated tumors in combination with radiotherapy to achieve a more complete destruction of deep-lying malignancies. With this strategy, photosensitizers, such as porphyrins, that have been abandoned owing to their unfavorable excitation range could be applicable as they can be now excited at 400 $\mathrm{nm}$ (Soret band) with a tenfold stronger absorption than the commonly used absorption band at $630 \mathrm{~nm}$. Given the paramagnetism of $\mathrm{Gd}^{3+}$ and the ease of including different lanthanides in the nanolipoparticles, MRI-guided X-ray triggered PDT therapies could be envisaged. The versatility and the widespread biomedical use of liponanoparticles, the possibility of image-guided therapies, and the unlimited tissue penetration depth thanks to X-ray excitation make this strategy particularly promising, enabling fundamentally new perspectives in photodynamic therapy.

\section{Acknowledgements}

Data collection was performed on the METROLOGY and DISCO beamline (using the Biology support lab) at Synchrotron SOLEIL, France. We thank Dr. Célia Bonnet for her help in the preparation of the micelles, and Dr Frédéric Jamme, Valérie Rouam and Paulo Da Silva for their help and technical support. We are grateful to the SOLEIL synchrotron for general facilities placed at our disposure. This research was supported by SOLEIL (No. 20100575) and the Regional Council of the Région Centre (convention SOLEIL / Région Centre 201100070573 - DEEP-PDT).

Electronic Supplementary Material: Supplementary material (preparation of the micelles, synchrotron $\mathrm{X}$-Ray irradiation setup, cell culture conditions and six supplementary figures) is available in the online version of this article at http:/ / dx.doi.org/10.1007/s12274-015-0747-5.

\section{References}

[1] Setua, S.; Menon, D.; Asok, A.; Nair S.; Koyakutty, M. Folate receptor targeted, rare-earth oxide nanocrystals for bi-modal fluorescence and magnetic imaging of cancer cells. Biomaterials 2010, 31, 714-729.

[2] Bünzli, J. C. G.; Piguet, C. Taking advantage of luminescent lanthanide ions. Chem. Soc. Rev. 2005, 34, 1048-1077.

[3] Bruchez, M.; Moronne, M.; Gin, P.; Weiss, S.; Alivisatos, A. P. Semiconductor nanocrystals as fluorescent biological labels. Science 1998, 281, 2013-2016.

[4] Chan, W. C. W.; Nie, S. M. Quantum dot bioconjugates for ultrasensitive nonisotopic detection. Science 1998, 281, 2016-2018.

[5] Delehanty, J. B.; Bradburne, C. E.; Susumu, K.; Boeneman, K.; Mei, B. C.; Farrell, D.; Blanco-Canosa, J. B.; Dawson, P. E.; Mattoussi, H.; Medintz, I. L. Spatiotemporal multicolor labeling of individual cells using peptide-functionalized quantum dots and mixed delivery techniques. J. Am. Chem. Soc. 2011, 133, 10482-10489.

[6] Pinaud, F.; Clarke, S.; Sittner, A.; Dahan, M. Probing cellular events, one quantum dot at a time. Nat. Methods 2010, 7, 275-285.

[7] Faulkner, S.; Pope, S. J. A.; Burton-Pye, B. P. Lanthanide complexes for luminescence imaging applications. Appl. Spectrosc. Rev. 2005, 40, 1-31.

[8] LaVan, D. A.; McGuire, T.; Langer, R. Small-scale systems for in vivo drug delivery. Nat. Biotechnol. 2003, 21, 1184-1191.

[9] Brigger, I.; Dubernet, C.; Couvreur, P. Nanoparticles in cancer therapy and diagnosis. Adv. Drug Delivery Rev. 2002, 54, 631-651.

[10] Xie, J.; Lee, S.; Chen, X. Y. Nanoparticle-based theranostic agents. Adv. Drug Delivery Rev. 2010, 62, 1064-1079.

[11] Caravan, P.; Ellison, J. J.; McMurry, T. J.; Lauffer, R. B. Gadolinium (III) chelates as MRI contrast agents: Structure, dynamics, and applications. Chem. Rev. 1999, 
99, 2293-2352.

[12] Zhou, Z. X.; Lu, Z. R. Gadolinium-based contrast agents for magnetic resonance cancer imaging. Wiley Interdiscip. Rev.: Nanomed. Nanobiotechnol. 2013, 5, 1-18.

[13] Merbach, A. S.; Helm, L. Toth, E. The Chemistry of Contrast Agents in Medical Magnetic Resonance Imaging; John Wiley \& Sons, Ltd: Chichester, West Sussex, UK, 2013.

[14] Maguire, J. A.; Zhu, Y. Boron and Gadolinium Neutron Capture Therapy for Cancer Treatment; World Scientific Pub. Co.: Hackensack, NJ, 2012.

[15] Eliseeva, S. V.; Bünzli, J. C. G. Lanthanide luminescence for functional materials and bio-sciences. Chem. Soc. Rev. 2010, 39, 189-227.

[16] Werts, M. H. V. Making sense of lanthanide luminescence. Sci. Prog. 2005, 88, 101-131.

[17] Urbain, G. La phosphorescence cathodique des terres rares. Ann. Chim. Phys. 1909, 8, 222-375.

[18] Kaščáková, S.; Giuliani, A.; Jamme, F.; Refregiers, M. Photodynamic Therapy. In Radiation Damage in Biomoleculare Systems; Springer: Dordrecht, Heidelberg, London, New York, 2012; pp 445-460.

[19] Roelants, M.; Lackner, B.; Waser, M.; Falk, H.; Agostinis, P.; Van Poppel, H.; de Witte, P. A. M. In vitro study of the phototoxicity of bathochromically-shifted hypericin derivatives. Photochem. Photobiol. Sci. 2009, 8, 822-829.

[20] Carpenter, S.; Fehr, M. J.; Kraus, G. A.; Petrich, J. W. Chemiluminescent activation of the antiviral activity of hypericin: A molecular flashlight. Proc. Natl. Acad. Sci. USA 1994, 91, 12273-12277.

[21] Wen, J.; Chowdhury, P.; Wills, N. J.; Wannemuehler, Y.; Park, J.; Kesavan, S.; Carpenter, S.; Kraus, G. A.; Petrich, J. W. Toward the molecular flashlight: Preparation, properties and photophysics of a hypericin-luciferin tethered molecule. Photochem. Photobiol. 2002, 76, $153-157$.

[22] Theodossiou, T.; Hothersall, J. S.; Woods, E. A.; Okkenhaug, K.; Jacobson, J.; MacRobert, A. J. Firefly luciferin-activated rose bengal: In vitro photodynamic therapy by intracellular chemiluminescence in transgenic
NIH 3 T3 cells. Cancer Res. 2003, 63, 1818-1821.

[23] Wang, F.; Zhang, Y.; Fan, X. P.; Wang, M. Q. Facile synthesis of water-soluble $\mathrm{LaF}_{3}: \mathrm{Ln}^{3+}$ nanocrystals. $J$. Mater. Chem. 2006, 16, 1031-1034.

[24] Liu, Y. F.; Chen, W.; Wang, S. P.; Joly, A. G.; Westcott, S.; Woo, B. K. X-ray luminescence of $\mathrm{LaF}_{3}: \mathrm{Tb}^{3+}$ and $\mathrm{LaF}_{3}: \mathrm{Ce}^{3+}, \mathrm{Tb}^{3+}$ water-soluble nanoparticles. J. Appl. Phys. 2008, 103, 063105.

[25] Liu, Y. F.; Chen, W.; Wang, S. P.; Joly, A. G. Investigation of water-soluble $\mathrm{X}$-ray luminescence nanoparticles for photodynamic activation. Appl. Phys. Lett. 2008, 92, 043901.

[26] Bulin, A. L.; Truillet, C.; Chouikrat, R.; Lux, F.; Frochot, C.; Amans, D.; Ledoux, G.; Tillement, O.; Perriat, P.; Barberi-Heyob, M. et. al. X-ray-induced singlet oxygen activation with nanoscintillator-coupled porphyins. $J$. Phys. Chem. C 2013, 117, 21583-21589.

[27] Ma, L.; Zou, X.; Chen, W. A new X-ray activated nanoparticle photosensitizer for cancer treatment. $J$. Biomed. Nanotechnol. 2014, 10, 1501-1508.

[28] Ma, L.; Zou, X. J.; Bui, B.; Chen, W.; Song, K. H.; Solberg, T. X-ray excited $\mathrm{ZnS}$ : $\mathrm{Cu}$, Co afterglow nanoparticles for photodynamic activation. Appl. Phys. Lett. 2014, 105, 013702.

[29] Bonnet, C. S.; Pellegatti, L.; Buron, F.; Shade, C. M.; Villette, S.; Kubíček, V.; Guillaumet, G.; Suzenet, F.; Petoud, S.; Tóth, É. Hydrophobic chromophore cargo in micellar structures: A different strategy to sensitize lanthanide cations. Chem. Commun. 2010, 46, 124-126.

[30] Bonnet, C. S.; Buron, F.; Caillé, F., Shade, C. M.; Drahoš, B.; Pellegatti, L.; Zhang, J.; Villette, S.; Helm, L.; Pichon, C. et. al. Pyridine-based lanthanide complexes combining MRI and NIR luminescence activities. Chem.-Eur. J. 2012, 18, 1419-1431.

[31] Chrysochoos, J. Fluorescence enhancement of $\mathrm{Eu}^{3+}$ by $\mathrm{Tb}^{3+}$ in dimethylsulfoxide (DMSO). J. Lumin. 1974, 9, 79-93.

[32] Jiao, H.; Zhang, N.; Jing, X. P.; Jiao, D. M. Influence of rare earth elements ( $\mathrm{Sc}, \mathrm{La} \mathrm{Gd}$ and $\mathrm{Lu}$ ) on the luminescent properties of green phosphor $\mathrm{Y}_{2} \mathrm{SiO}_{5}: \mathrm{Ce}, \mathrm{Tb}$. 
Opt. Mater. (Amst). 2007, 29, 1023-1028.

[33] Armelao, L.; Heigl, F.; Jürgensen, A.; Blyth, R. I. R.; Regier, T.; Zhou, X. T.; Sham, T. K. X-ray excited optical luminescence studies of $\mathrm{ZnO}$ and Eu-doped $\mathrm{ZnO}$ nanostructures. J. Phys. Chem. C 2007, 111, 10194-10200.

[34] Trans-1-(2'-methoxyvinyl)pyrene. http://products.invitrogen.com/ivgn/product/M7913 (accessed Dec 24, 2014).

[35] Kascakova, S.; Refregiers, M.; Jancura, D.; Sureau, F.; Maurizot, J. C.; Miskovsky, P. High level of low-density lipoprotein receptors enhance hypericin uptake by U-87 MG cells in the presence of LDL. Photochem. Photobiol. 2005, 81, 1395-1403.

[36] Giuliani, A.; Jamme, F.; Rouam, V.; Wien, F.; Giorgetta, J. L.; Lagarde, B.; Chubar, O.; Bac, S.; Yao, I.; Rey, S. et al. DISCO: A low-energy multipurpose beamline at synchrotron SOLEIL. J. Synchrotron Radiat. 2009, 16,
$835-841$.

[37] Jamme, F.; Villette, S.; Giuliani, A.; Rouam, V.; Wien, F.; Lagarde, B.; Réfrégiers, M. Synchrotron UV fluorescence microscopy uncovers new probes in cells and tissues. Microsc. Microanal. 2010, 16, 507-514.

[38] Edelstein, A.; Amodaj, N.; Hoover, K.; Vale, R.; Stuurman, N. Computer control of microscopes using micromanager. Curr. Protoc. Mol. Biol. 2010, 92, 14.20.1-14.20.17. 


\title{
Electronic Supplementary Material
}

\section{X-ray-induced radiophotodynamic therapy (RPDT) using lanthanide micelles: Beyond depth limitations}

\author{
Slávka Kaščáková ${ }^{1,2}$, Alexandre Giuliani ${ }^{1,3}$, Sara Lacerda ${ }^{4}$, Agnès Pallier $^{4}$, Pascal Mercère ${ }^{1}$, Éva Tóth $^{4}$, Matthieu \\ Réfrégiers ${ }^{1}(\bowtie)$ \\ ${ }^{1}$ Synchrotron SOLEIL, L'Orme des Merisiers, 91190 Gif-sur-Yvette, France \\ ${ }^{2}$ Université Paris-Sud XI, UMR-785, 94807 Villejuif, France \\ ${ }^{3}$ INRA, UAR1008 Caractérisation et Élaboration des Produits Issus de l'Agriculture, 44316 Nantes, France \\ ${ }^{4}$ Centre de Biophysique Moléculaire, CNRS, Rue Charles Sadron, 45071 Orléans Cedex 2, France
}

Supporting information to DOI 10.1007/s12274-015-0747-5

Preparation of GdEuC12 micelles: The GdEuC12 micelles were prepared according to Bonnet et al [S1]. Briefly, a $6.95 \mathrm{mM}$ aqueous solution of the C12 ligand was prepared, and an equimolar amount of lanthanide chloride was added in a molar ratio of $90 \% \mathrm{GdCl}_{3}$ and $10 \% \mathrm{EuCl}_{3}$. The solution was allowed to react at $333 \mathrm{~K}$ by regularly controlling the $\mathrm{pH}$, adjusting the $\mathrm{pH}$ with $\mathrm{KOH}$ when necessary. The absence of free metal was verified by xylenol orange colorimetric assay. The micelle size was verified by dynamic light scattering (DLS), measured with Mavern Zetasizer Nano Series equipment at $20.0^{\circ} \mathrm{C}$, revealing an average diameter of $4.6 \mathrm{~nm}$ with a PDI of 0.6.

Preparation of Hyp-GdEuC12 micelles: Stock solutions of Hyp (1.98 mM and $0.1 \mathrm{mM})$ were prepared by dissolving Hyp in 100\% dimethyl sulfoxide (DMSO), and the solutions were stored in the dark at $4{ }^{\circ} \mathrm{C}$ until used. The Hyp-micelles were prepared by mixing a GdEuC12 micelle solution with Hyp. Four Hyp-GdEuC12 solutions were prepared: The concentration of $\mathrm{GdEuC}_{12}$ micelles was kept constant $(6.95 \mathrm{mM})$, while the concentration of Hyp varied $(\mathrm{c}=0.2,4,20$, and $60 \mu \mathrm{M})$. The total content of DMSO in the final micellar solution did not exceed 3\%. After mixing, the micelle solutions were sonicated in the dark for $10 \mathrm{~min}$ at $37^{\circ} \mathrm{C}$. The incorporation of Hyp into the hydrophobic core of micelle was verified by the Hyp absorption and fluorescence measurements, as indicated in our previous study [S2]. The prepared Hyp-GdEuC $\mathrm{C}_{12}$ micelles were then stored in the dark at $4^{\circ} \mathrm{C}$ for at least $24 \mathrm{~h}$ before X-ray irradiation. The DLS measurements were performed to verify the existence of the micelles. The critical micellar concentration ( $\mathrm{cmc}$ ) was determined for the Hyp-GdEuC12 micelles by a previously described relaxometric method [S3]. Based on the measurement of proton relaxation rates at variable concentrations, the $\mathrm{cmc}$ was found to be $1.6 \mathrm{mM}(\mathrm{LnL})$ in aqueous solution containing 3\% DMSO $([\mathrm{Hyp}]=0.008[\mathrm{GdL}])$. Using the same relaxometric method, we 
found that in the cell culture medium (described below), aggregates are present above the $0.1 \mathrm{mM}$ concentration of LnC12 ([Hyp] = 0.008[GdL]).

The incorporation of Hyp was also demonstrated by recording the Eu luminescence emission spectrum following excitation at $550 \mathrm{~nm}$, corresponding to Hyp excitation (Fig. S4). The excitation spectrum recorded (for Eu emission at $619 \mathrm{~nm}$ ) overlaps with the absorption spectrum of Hyp, showing that Eu luminescence results from sensibilization via Hyp as an antenna. This is only possible if Hyp and Eu are in close proximity, i.e., within the same micellar aggregate [S1, S4].

X-ray irradiation and experimental set-up: Experiments were performed on the METROLOGY and Tests beamline of the Synchrotron SOLEIL using the multiple bunch mode (400-mA ring current) of the machine. The sample was placed $32 \mathrm{~m}$ from the bending magnet source, after a $150-\mu \mathrm{m}$-thick Beryllium window and a 1.45-m air path. The white beam radiation delivered by the beamline was adjusted to a transverse size of 13 (Hor.) x 2 (Vert.) $\mathrm{mm}^{2}$ at the sample position. Figure S5 gives the simulated X-ray beam spectrum for our experimental setup as implemented on the beamline. The integrated flux on the sample was $10^{15}$ photons/s. Several motorized translation stages allowed for precise sample alignment within the beam path. The sample holder was also equipped with a quartz collimating lens coupled to a $600-\mu \mathrm{m}$ optical fiber (Ocean Optics, France) to collect (perpendicular to the X-ray beam) the UV fluorescence emitted by the sample. A Maya2000 Pro (Ocean Optics, France) spectrometer (covering the 175-1100 nm wavelength range) was finally used for spectral analysis of the sample fluorescence during X-ray irradiation. To increase the signal-to-noise (S/N) ratio, all spectra were averaged over 10 acquisitions of $30 \mathrm{~s}$ integration time.

Detection of singlet oxygen production after X-ray irradiation of Hyp-GdEuC12 micelle solutions using mass spectrometry: We have chosen methoxyvinylpyrene (MVP) because it is highly specific for singlet oxygen and does not react with other activated oxygen species such as hydroxyl radicals, superoxide, or hydrogen peroxide [S5]. Although MVP is used as chemiluminescence probe of singlet oxygen, the high concentrations of photosensitizer present in our micelles as well as changes of luminescence properties of $\mathrm{GdEu}$ micelles after X-ray irradiation made the detection of singlet oxygen by chemiluminescence of MVP difficult. The mechanism of MVP reaction with singlet oxygen is very well known: The reaction of MVP with singlet oxygen decomposes the MVP molecule into 1-pyrenecarboxaldehyde. Based on the known molecular weight of 1-pyrenecarboxaldehyde $(M w=230.6)$ and MVP $(M w=258.31)$, we decided to use mass spectrometry to detect singlet oxygen production. The validation of mass spectrometry as a tool to investigate singlet oxygen production is part of Fig. S6. The singlet oxygen production was monitored by mass spectrometry after polychromatic X-ray irradiation of GdEuC12 $(c \mathrm{Ln}=6.95 \mathrm{mM})$ micelle-Hyp solutions $(c=0.5,3,7,10,12$, and $20 \mu \mathrm{M})$ with singlet oxygen probe MVP $(c=1 \mu \mathrm{M})$. After mixing $1 \mu \mathrm{M} \mathrm{MVP}$ with GdEuC12 micelle-Hyp complexes, the solution was divided into two aliquots: The first was injected into the mass spectrometer to measure the relative molecular weights of the singlet oxygen probe in the non-irradiated sample, and the second was irradiated for $6 \mathrm{~s}$ with the polychromatic synchrotron beam. After irradiation, the sample was inserted into the mass spectrometer, and the intensities of molecular masses corresponding to MVP and 1-pyrenecarboxaldehyde were measured. All measurements were done three times in the absence of visible light.

To validate the use of mass spectrometry as a tool for the detection of singlet oxygen species, the results have been compared with gold standard method, fluorescence spectroscopy, using the singlet oxygen sensitive probe MVP. We have chosen water-soluble Al(III) phthalocyanine chloride tetrasulfonic acid for the

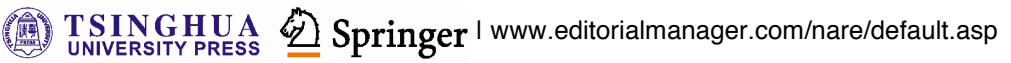


photosensitizer. A solution of this photosensitizer at concentration $c=0.5 \mu \mathrm{M}$ has been prepared in phosphate-buffered saline (PBS) at $\mathrm{pH}=7.4$, followed by mixing with the singlet oxygen probe MVP ( $\mathrm{c}=3$ $\mu \mathrm{M})$. To produce singlet oxygen species, the solution, which was contained in a quartz cuvette with a $1-\mathrm{cm}$ pathlength, was irradiated with a He-Ne laser at 633-nm wavelength. During irradiation, the solution was stirred with a magnetic stirrer. After each irradiation dose, the aliquot of the irradiated solution was analyzed by mass spectroscopy and/or by fluorescence spectroscopy. The fluorescence of the MVP probe was monitored at $\lambda_{\mathrm{mis}}=467 \mathrm{~nm}$ (excitation wavelength at $\lambda_{\mathrm{acc}}=400 \mathrm{~nm}$ ). Figure $\mathrm{S6}$ shows the correlation of singlet oxygen production detected by both methods. While Fig. S6(a) demonstrates the singlet oxygen production measured by fluorescence spectroscopy, Figure S6(b) shows the singlet oxygen production (within the same sample of irradiated photosensitizer) measured by mass spectroscopy. The error bars within Fig. S6(b) came from two injections of the same sample. A comparison of both graphs in Fig. S6 shows the same profile, which validates the use of mass spectrometry as a tool for detecting singlet oxygen species.

Cell culture and conditions of incubations: The HeLa human cells were routinely cultured as monolayer and were grown in Dulbecco's modified Eagle's medium (DMEM) containing L-glutamine (862 $\left.\mathrm{mg}^{-\mathrm{L}^{-1}}\right)$, sodium pyruvate $\left(110 \mathrm{mg} \cdot \mathrm{L}^{-1}\right)$, and glucose $\left(4500 \mathrm{mg} \cdot \mathrm{L}^{-1}\right)$, supplemented with $10 \%$ fetal calf serum (FCS), penicillin $\left(50 \mu \mathrm{g} \cdot \mathrm{mL}^{-1}\right)$, and streptomycin $\left(50 \mu \mathrm{g} \cdot \mathrm{mL}^{-1}\right)$. All chemicals were obtained from Gibco-Invitrogen. The cells were maintained at $37{ }^{\circ} \mathrm{C}$ in a humidified atmosphere of $5 \% \mathrm{CO}_{2}$. For detecting Hyp-GdEuC12 micelles in the cells, both control and treated cells were processed in a similar way. The cells were seeded on quartz slides (deposited in $35 \times 10 \mathrm{~mm}$ Petri dishes) to reach confluency after $48 \mathrm{~h}$ of incubation at $37^{\circ} \mathrm{C}$ under an atmosphere of $95 \%$ air $/ 5 \% \mathrm{CO}_{2}$. The medium was removed and fresh growth medium (supplemented with 5\% FCS, glutamine, penicillin, and streptomycin) was added to each flask. In the case of treated cells, the medium was mixed with Hyp alone $(4 \mu \mathrm{M})$, with the GdEuC12 micelles alone $(500 \mu \mathrm{M})$, and with Hyp-GdEuC12 micelles (500 $\mu \mathrm{M}$ Ln; $4 \mu \mathrm{M})$. The medium was added to the Petri dishes, which were incubated with cells for $4 \mathrm{~h}$. After a given period of time, the cells were washed twice with PBS and were fixed with $4 \%$ paraformaldehyde.

\section{Supplementary figures}




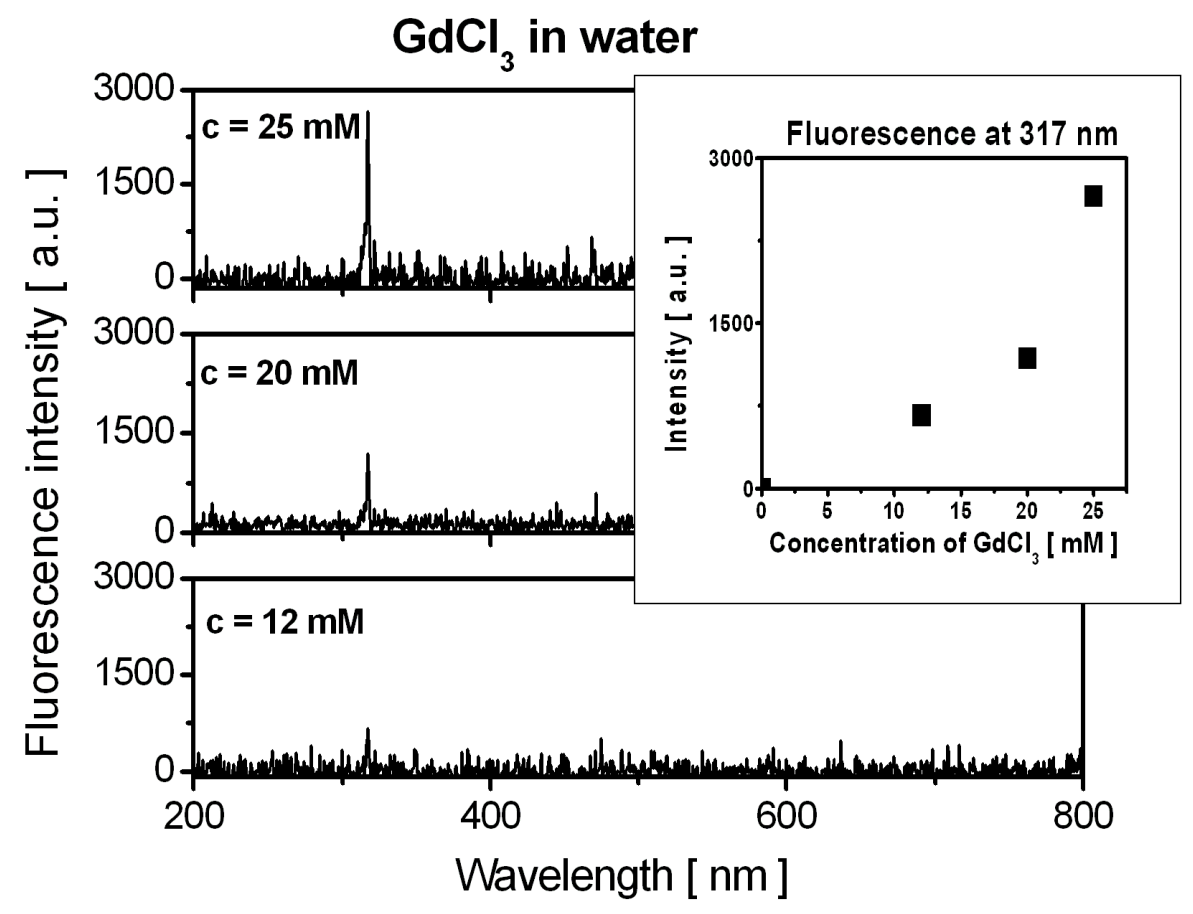

Figure S1 Concentration dependence of the X-ray-excited luminescence of $\mathrm{GdCl}_{3}$ in distilled water.

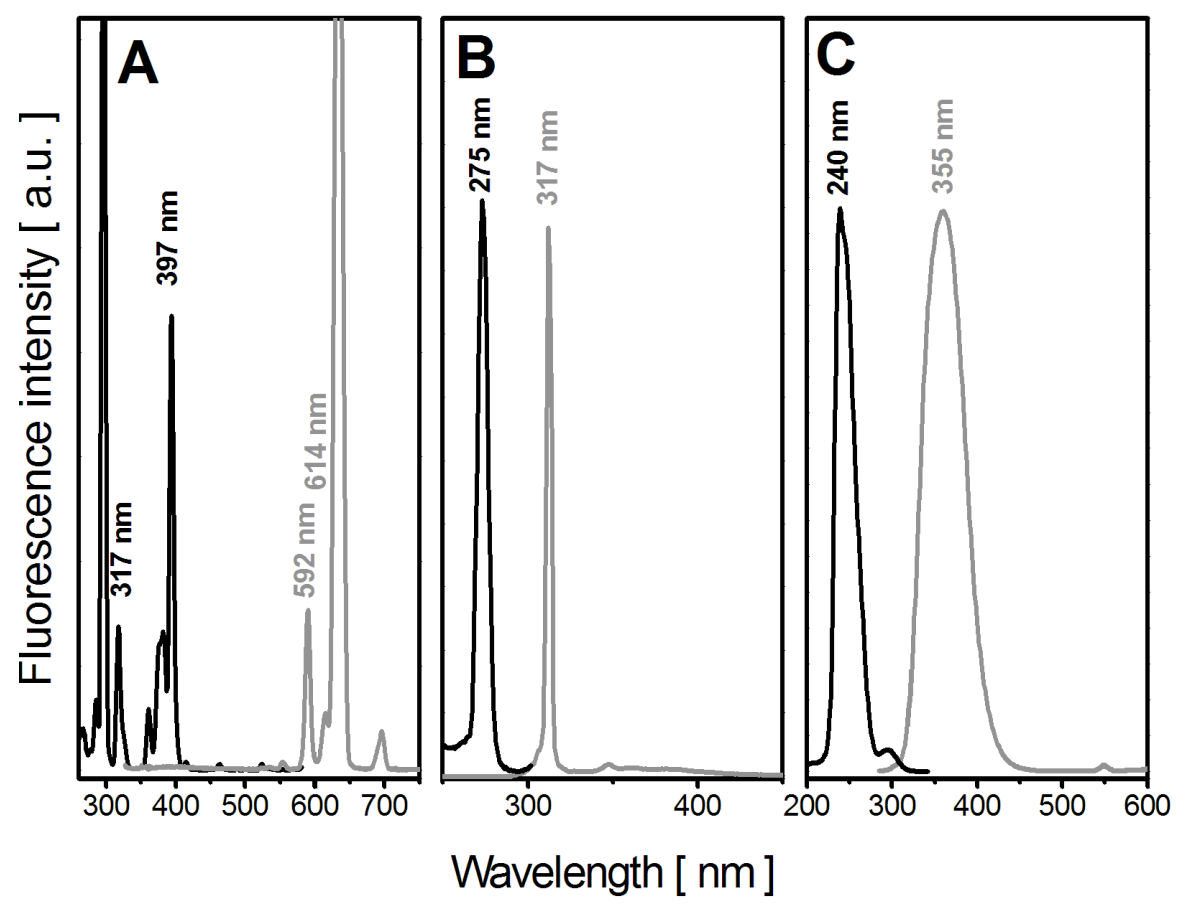

Figure S2 Photoluminescence spectra of lanthanides in aqueous solution: (a) $\mathrm{EuCl}_{3}(c=96 \mathrm{mM})$ emission spectrum at $\lambda_{\mathrm{ecc}}=317 \mathrm{~nm}$ (grey) and excitation spectrum at $\lambda_{\text {emis }}=592 \mathrm{~nm}$ (black); (b) $\mathrm{GdCl}_{3}(c=12.7 \mathrm{mM})$ emission spectrum at $\lambda_{\mathrm{ecc}}=275 \mathrm{~nm}$ (grey) and excitation spectrum at $\lambda_{\text {enis }}=317 \mathrm{~nm}$ (black); (c) $\mathrm{CeCl}_{3}(c=0.883$ $\mathrm{mM}$ ) emission spectrum at $\lambda_{\mathrm{ex}}=240 \mathrm{~nm}$ (grey) and excitation spectrum at $\lambda_{\mathrm{enis}}=355 \mathrm{~nm}$ (black).

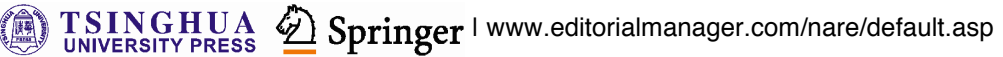




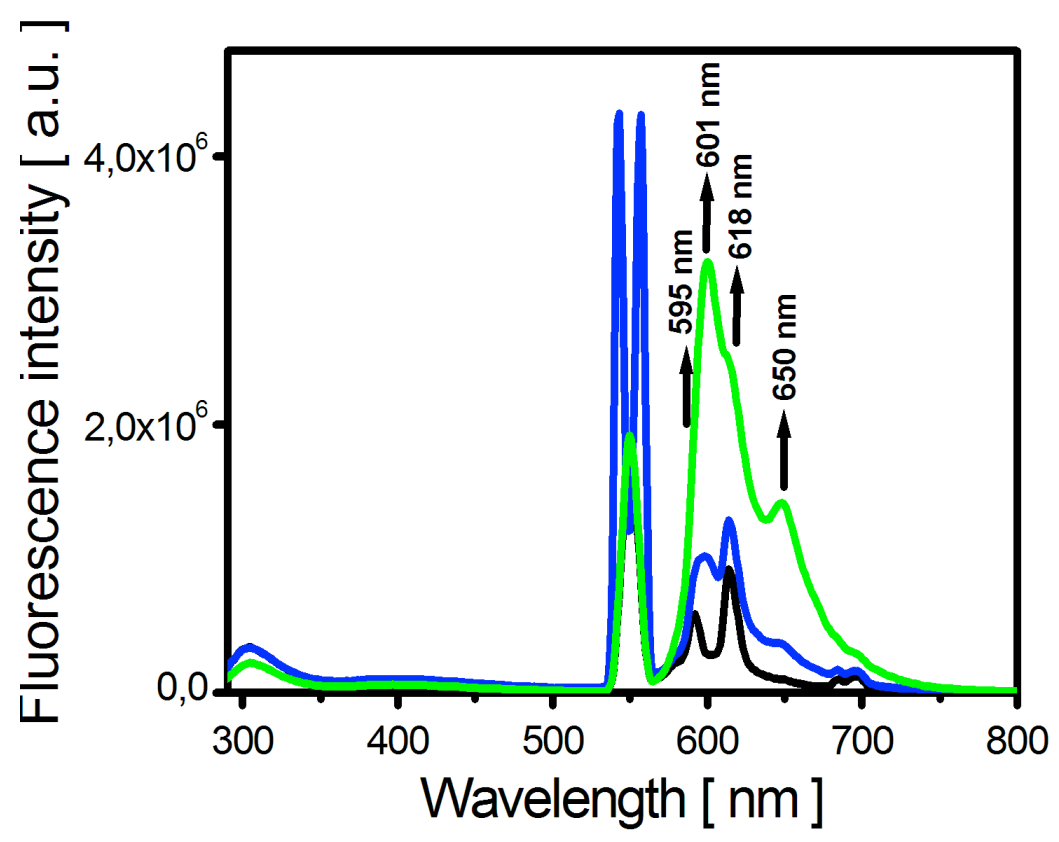

Figure S3 Photoluminescence spectra of Hyp-GdEuC12 micelles in the presence of different concentrations of Hyp at $\lambda_{\mathrm{ac}}=275 \mathrm{~nm}$ : GdEuC12 micelles without Hyp (black); Hyp-GdEuC12 micelles with $3 \mu \mathrm{M}$ (blue); and Hyp-GdEuC12 micelles with $20 \mu \mathrm{M}$ Hyp (green). The peak at $550 \mathrm{~nm}$ corresponds to the second harmonic excitation. The peaks at 601 and $650 \mathrm{~nm}$ are the peaks of Hyp, while the peaks at $595 \mathrm{~nm}$ and 618 nm correspond to Eu fluorescence.

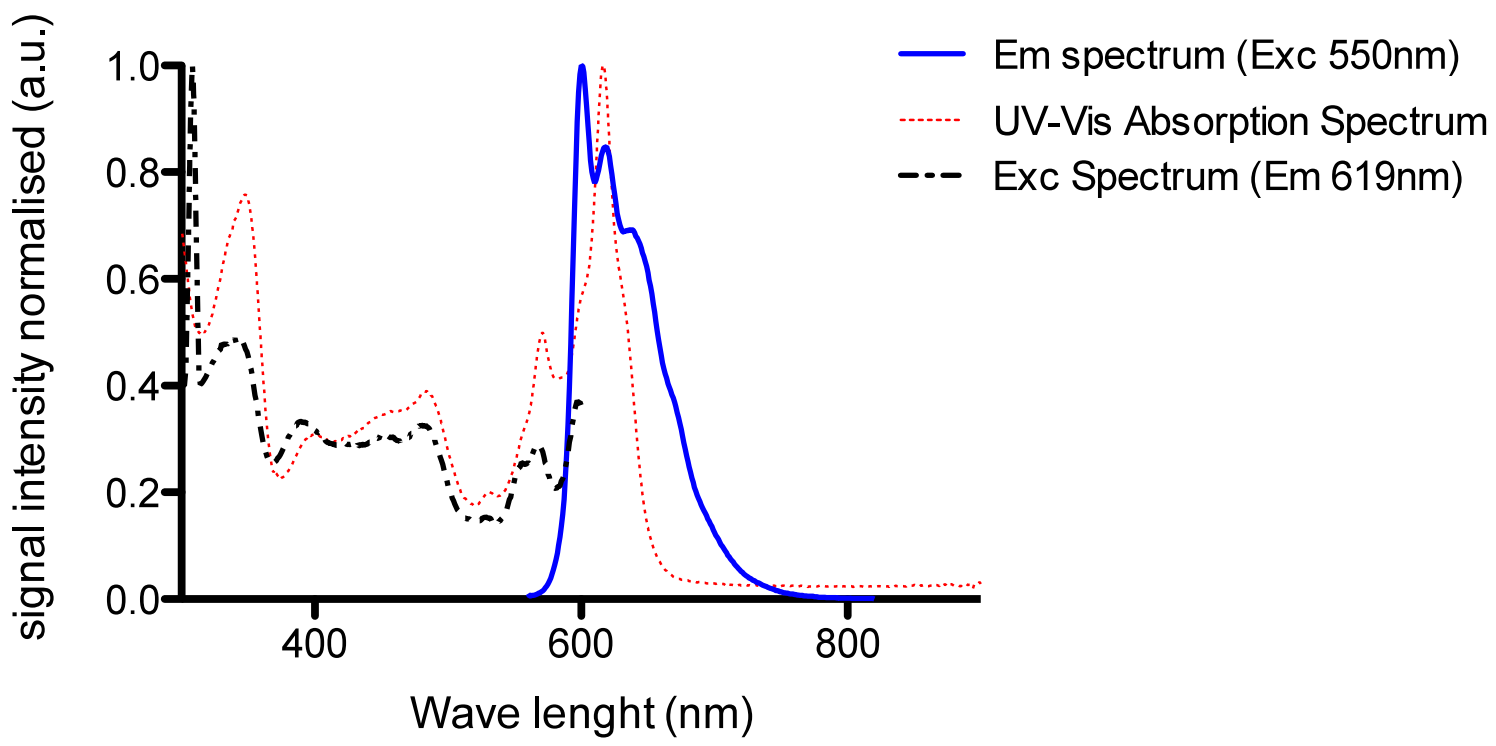

Figure S4 Absorption, normalized emission $\left(\lambda_{\text {ex }}=550 \mathrm{~nm}\right)$, and excitation $\left(\lambda_{\text {enis }}=619\right.$ nm $)$ spectra of GdEuLC12 micelles (6.95 mM LnL) containing $60 \mu \mathrm{M}$ Hyp. 


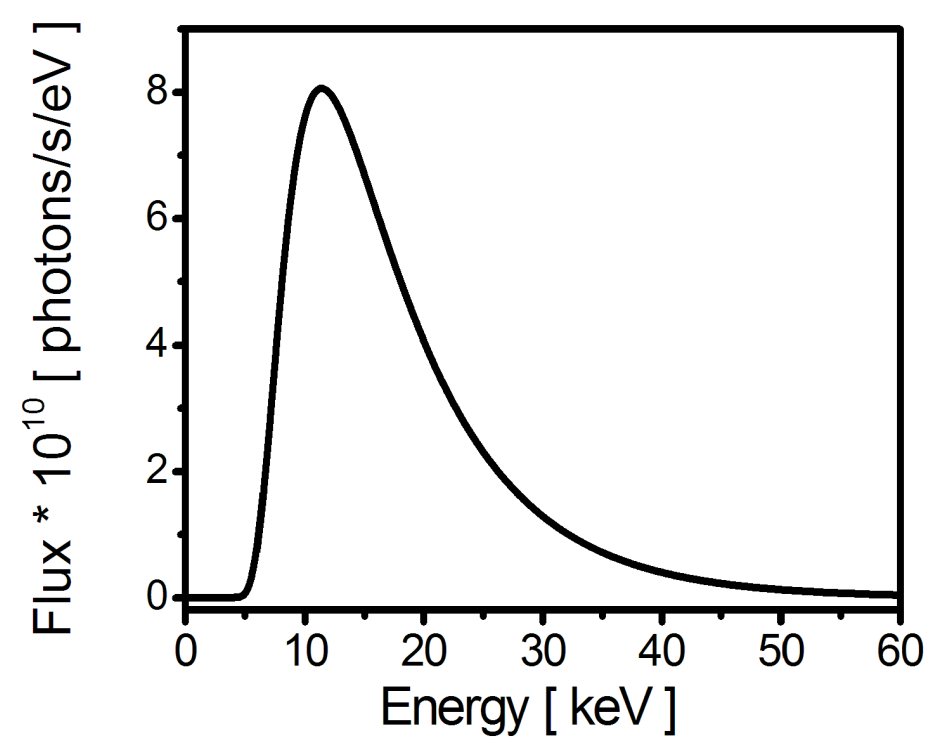

Figure S5 The X-ray beam spectrum impinging on the sample in the configuration of our experiment on the METROLOGY beamline.

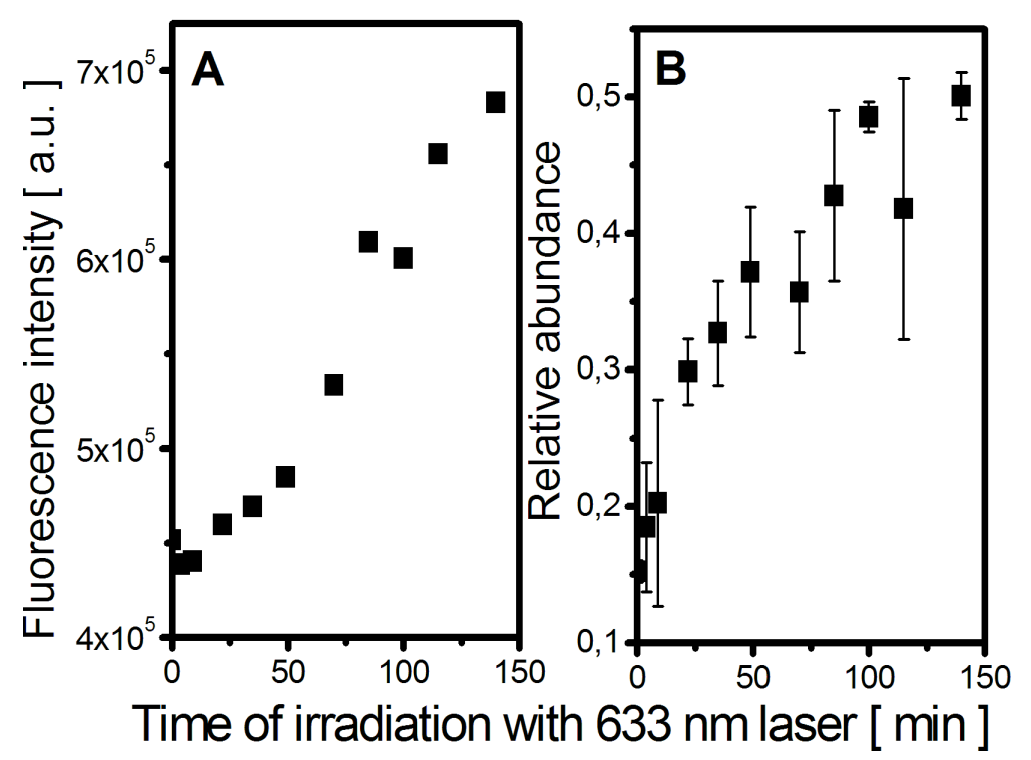

Figure S6 (a) Singlet oxygen production of Al(III) phthalocyanine chloride tetrasulfonic acid in PBS with pH $=7.4$ measured by fluorescence spectroscopy. The photosensitizer was irradiated with a 633-nm wavelength laser. The production of singlet oxygen was measured by an MVP probe. The MVP probe only fluoresces at $467 \mathrm{~nm}$ in presence of singlet oxygen. (b) Singlet oxygen production of Al(III) phthalocyanine chloride tetrasulfonic acid in PBS with $\mathrm{pH}=7.4$ measured by mass spectrometry. The relative abundance of 1-pyrenecarboxaldehyde, a singlet oxygen indicator, after irradiation of $\mathrm{Al}(\mathrm{III})$ Phthalocyanine Chloride Tetrasulfonic Acid in PBS pH = 7.4. 


\section{Reference}

[S1] Bonnet, C. S.; Pellegatti, L.; Buron, F.; Shade, C. M.; Villette, S.; Kubíček, V.; Guillaumet, G.; Suzenet, F.; Petoud, S.; Tóth, É. Hydrophobic chromophore cargo in micellar structures: A different strategy to sensitize lanthanide cations. Chem. Commun. 2010, 46, $124-126$.

[S2] Kascakova, S.; Refregiers, M.; Jancura, D.; Sureau, F.; Maurizot, J. C.; Miskovsky, P. High level of low-density lipoprotein receptors enhance hypericin uptake by U-87 MG cells in the presence of LDL. Photochem. Photobiol. 2005, 81, 1395-1403.

[S3] Nicolle, G. M.; Tóth, E.; Eisenwiener, K. P.; Mäcke, H. R.; Merbach, A. E. From monomers to micelles: Investigation of the parameters influencing proton relaxivity. J. Biol. Inorg. Chem. 2002, 7, 757-769.

[S4] Lacerda, S.; Bonnet, C. S.; Pallier, A.; Villette, S.; Foucher, F.; Westall, F.; Buron, F.; Suzenet, F.; Pichon, C.; Petoud, S.; Tóth, É. Lanthanide-based, near-infrared luminescent and magnetic lipoparticles: Monitoring particle integrity. Small 2013, 9 , 2662-2666.

[S5] Trans-1-(2'-methoxyvinyl)pyrene. http: / / products.invitrogen.com/ivgn/product/M7913 (accessed Dec 24, 2014).

\footnotetext{
Address correspondence to Matthieu Réfrégiers, matthieu.refregiers@synchrotron-soleil.fr
} 